\title{
Development of a Conceptual Model of Chronic Lymphocytic Leukemia to Better Understand the Patient Experience
}

\author{
Daniel Eek ${ }^{1} \cdot$ Matthew Blowfield ${ }^{2} \cdot{\text { Calvin } \mathrm{Krogh}^{3} \cdot \text { Helena Chung }}^{4} \cdot$ Toby A. Eyre $^{5}$
}

Published online: 18 August 2020

(c) The Author(s) 2020

\begin{abstract}
Background and Objective Understanding the patient experience is important for identifying the unmet need in chronic lymphocytic leukemia. The current study aimed to develop a comprehensive chronic lymphocytic leukemia conceptual model. Methods The conceptual model was based on literature searches, review of chronic lymphocytic leukemia patient blogs/ forums, and interviews with five expert clinicians, with 20 patients who received at most one treatment (first line) for their chronic lymphocytic leukemia, and with 20 patients with relapsed or refractory chronic lymphocytic leukemia. De-identified interviews were transcribed, coded, and evaluated using qualitative data analysis software.

Results Thirty-five prevalent chronic lymphocytic leukemia-related symptom and impact concepts were identified from literature searches, patient blogs/forums, and clinician interviews. Patient interviews confirmed the identified concepts and revealed five additional concepts. Fatigue-related sub-components were identified from how patients described their fatigue, covering symptoms (tiredness/need for sleep, lack of energy, weakness, cognitive fatigue), and impacts (decreased ability to maintain their social, familial, or professional role, decreased physical functioning, frustration). Three versions of the conceptual model were created: an overall model with all concepts; a model highlighting the most prominent concepts in first line; and a model highlighting the most prominent concepts in relapsed or refractory disease. Prominent concepts in both first line and relapsed or refractory disease were fatigue-related symptoms and impacts, muscle/joint aches, night sweats, bruising, fever, recurrent infections/illness, insomnia, decreased cognitive/emotional functioning, anxiety/worry, stress, depression, financial difficulty, and fear of death. Dyspnea and cough were prominent in first line only, and enlarged lymph nodes, headaches, pain/discomfort, weight loss, nausea/vomiting, and infusion reactions were prominent in relapsed or refractory disease only.

Conclusions The results show that fatigue is a dominant issue affecting patients with chronic lymphocytic leukemia. The three versions of the conceptual model can help researchers to understand patients' unmet needs and guide the patient-reported outcome strategy for clinical trials.
\end{abstract}

Electronic supplementary material The online version of this article (https://doi.org/10.1007/s40271-020-00440-9) contains supplementary material, which is available to authorized users.

Daniel Eek

daniel.eek@astrazeneca.com

1 AstraZeneca Gothenburg, Pepparedsleden 1, SE 43183 Mölndal, Sweden

2 IQVIA, Reading, UK

3 IQVIA, Cambridge, MA, USA

4 AstraZeneca LP, Gaithersburg, MD, USA

5 Oxford Cancer and Haematology Centre, Churchill Hospital, Oxford, UK

\section{Introduction}

Chronic lymphocytic leukemia (CLL) is a hematological malignancy that progresses slowly with a typically long natural history. In patients with CLL, abnormally proliferating lymphocytes accumulate in the blood, bone marrow, and lymphatic tissues, increasing the occurrence of infections, anemia, and bleeding [1, 2]. Early signs and symptoms of CLL are generally minimal, and the disease is often diagnosed following an incidental finding of lymphocytosis on a routine blood-cell count [3].

The chronic nature of CLL often requires long-term treatment with the goal of providing lasting benefits with minimal side effects [4-8]. At the early disease stage, symptom burden and risk of progression may be low, 


\section{Key Points for Decision Makers}

The symptoms, signs and impacts of chronic lymphocytic leukemia were identified by a literature review, interviews with hematologists, and qualitative concept elicitation interviews with patients who either had received at most one treatment for their chronic lymphocytic leukemia or had relapsed or refractory disease.

The most prominent issues mentioned by patients were fatigue-related symptoms and impacts, muscle/joint aches, night sweats, bruising, fever, recurrent infections/illness, insomnia, decreased cognitive/emotional functioning, anxiety/worry, stress, depression, financial difficulty, and fear of death.

Three versions of a conceptual model were developed that can help inform the selection and validation of patient-reported outcome instruments to evaluate new treatments for chronic lymphocytic leukemia.

and patients may be monitored without therapy ("watch and wait" or "active observation") [2]. Understanding how patients experience and are affected by CLL and its treatment is required for evaluating the overall benefit of therapy, both in the clinical trial setting and in routine clinical care [9]. Patient self-reporting of symptoms as part of routine oncology care may enhance symptom management, care outcomes, patient engagement, and healthrelated quality of life [10]. Obtaining information directly from patients about living with CLL is also essential for developing a patient-reported outcome (PRO) strategy for clinical trials [11-13]. PROs are often included in randomized clinical trials in oncology, at times as secondary or exploratory endpoints $[14,15]$. PRO results from trials can support labeling claims, healthcare policy, and clinical decision making $[16,17]$.

Qualitative patient interviews form the basis of developing and establishing content validity of PRO instruments, and ensure that the instrument captures all signs, symptoms, and impacts relevant to the target patient population. However, little has been published about the patient experience in CLL [18] and, historically, PROs have typically not been captured in pivotal CLL clinical trials [9], although there are a few recent exceptions [19-22]. The current qualitative research was undertaken to fill this gap with the aim of developing a comprehensive conceptual model of CLL with input from clinicians and patients, to help researchers to understand patients' unmet needs and direct future advancements in the field.

\section{Methods}

A draft conceptual model was constructed from a review of the literature and patient blogs/forums and was refined using information sourced from interviews with expert clinicians. The conceptual model was further refined and finalized after qualitative concept elicitation interviews with a representative cohort of patients either with at most one treatment (first-line [1L]) for their CLL or with relapsed or refractory (R/R) CLL. The methodological approach for this work was a qualitative content analysis.

\subsection{Review of Literature, Patient Blogs, and Forums}

Published information on the signs, symptoms, and impacts of CLL was identified by a targeted search of the literature in PubMed, Cochrane Library, and PsychINFO, conducted in September 2017, and a search of CLL patient blogs and forums (listed in the Electronic Supplementary Material [ESM] 1), conducted in October 2017. In addition, data on CLL treatment-related symptoms, and adverse event details for Bruton tyrosine kinase inhibitor, anti-CD20 monoclonal antibody, and chemotherapy treatments were reviewed for symptoms and impacts experienced by patients with CLL. All searches were conducted by IQVIA researchers (O. Meyers, C. Krogh, S. Lee).

For the literature searches, the following search string was used: ("CLL" OR "chronic lymphocytic leukaemia" OR "chronic lymphocytic leukemia") AND ("signs" OR "symptoms" OR "clinical manifestations" OR "patient reported outcomes" OR "PROs" OR "QoL" OR "HRQoL" OR "HRQL" OR "quality of life" OR "conceptual model" OR "disease model"). Abstracts were screened for key information about Participants, Interventions, Comparators, Outcomes and Study design ("PICOS" criteria) [23], and fulltext articles describing signs, symptoms, or impacts of CLL were reviewed. For the patient blogs and forums, identified concepts were considered prevalent if they were mentioned by four or more of the sites. A draft conceptual model was constructed based on the review of the literature and patient blogs/forums.

\subsection{Interviews with Expert Clinicians}

Telephone interviews were conducted with five expert hematologists practicing at prominent cancer research and clinical treatment centers in the USA (Mayo Clinic; Memorial Sloan Kettering Cancer Center; Willamette Valley Cancer Institute/US Oncology Network) or the UK (Leeds Teaching Hospital; Oxford University Hospital). Clinicians were contacted by the recruiting agency via phone or e-mail to determine willingness to participate. Purposive sampling 
was used to select the participants. All participants had a minimum of 5 years' experience in treating patients with CLL, had treated a minimum of ten patients with CLL each month, and spent more than $50 \%$ of their time in patient care. Consent for the clinician interviews was captured by the recruiting agency who sought out the clinicians and was obtained via screener information. There were no dropouts among clinicians who agreed to participate.

The interviews were conducted by trained interviewers experienced in interviews across a wide range of therapeutic areas (two were male, one was female; O. Meyers, C. Krogh, S. Lee; researchers, IQVIA). There was no relationship between the interviewers and clinicians prior to study commencement. No non-participants were present at the interviews. The interviewer introduced themselves, provided a study overview, and reiterated the study objectives. The interviews were conducted using a standardized semistructured interview guide with open-ended and prompted questions to explore the symptoms and impacts that the clinicians observed in their patients with CLL. In addition, input was sought on the draft conceptual model that was developed based on the review of the literature and patient blogs/forums. Interviews were recorded with the participants' permission and interviewers took informal notes to keep track of the conversation topic at hand. Each interview lasted approximately 60 minutes. Transcripts were not returned for comment or correction and no repeat interviews were conducted. Clinician interview data were analyzed descriptively. Based on IQVIA expertise, a sample size of five experts is sufficient to achieve saturation of findings. The draft conceptual model was refined in light of clinician feedback.

\subsection{Qualitative Patient Interviews}

Qualitative concept elicitation interviews were conducted in accordance with recommendations provided by the International Society for Pharmacoeconomics and Outcomes Research PRO Good Research Practices Task Force [24]. The patient interviews were approved by the New England Institutional Review Board. Patients with CLL in the $1 \mathrm{~L}$ setting (1L CLL), or in the R/R setting (R/R CLL) were recruited in the USA between May and August 2018. To be eligible, patients had to be aged 18 years or older, have a diagnosis of CLL with a self-reported Eastern Cooperative Oncology Group performance status of 0 , 1 , or 2 (i.e., still ambulatory and capable of all self-care) and be proficient in English. Patients in the R/R CLL group had to have received two or more therapies specifically to treat CLL. To ensure all patients could contribute to the understanding of the signs, symptoms, and impacts of CLL, included patients had to have experienced at least one of the following constitutional symptoms of the disease in the past week: fatigue; weight loss; fever; or night sweats.

Potential participants were identified via a patient advocacy organization (CLL Society; https://cllsociety.org) and two market research firms (Liberating Research and Rare Patient Voice) using purposive sampling, and were contacted by e-mail and telephone about study details and participation. Patients identified via the patient advocacy organization had completed an online screener on the organization's website or social media platforms. Patients identified via the market research firms had previously consented to be contacted regarding research participation opportunities. Informed consent was obtained by the recruiting agencies via an online form completed by patients before the interview and was confirmed at the start of each interview. Patients were asked to provide proof of diagnosis and disease staging by sending a form with this information to their clinician for certification, although this was not a requirement for participation. There were no dropouts amongst patients who agreed to participate.

All participants were interviewed by telephone using a standardized semi-structured interview guide with openended and prompted questions to explore patients' experiences with their CLL. Each interview lasted approximately 60-75 min, and started with the interviewer introducing themselves, providing a study overview, and reiterating the study objectives. Interviews were conducted by trained interviewers (two were male, one was female; O. Meyers, C. Krogh, S. Lee; researchers IQVIA) experienced in individual patient concept elicitation and cognitive debriefing interviews across a wide range of therapeutic areas. There was no relationship between interviewers and patients prior to study commencement. No non-participants were present at the interviews. Ahead of the patient interviews, the interviewers conducted either one or two pilot interviews with patients monitored by an IQVIA supervisor, to ensure consistency between interviewers and suitability of the interview guide.

As part of the open-ended questioning, patients were asked about their first experience of CLL, how their experience may have changed over time, and current symptoms, signs, and impacts of the disease and its treatments. Concepts mentioned during the open-ended questioning were coded as "spontaneous". Patients were then prompted about specific concepts not mentioned in the conceptual model; any that the patient reported in this part of the questioning were coded as "probed". To explore whether concepts were related to the disease, its treatment, or both, interviewers probed patients on whether the concept was experienced before, during, or after treatment. For each concept identified, interviewers asked patients to rate how disturbing the symptom, sign, or impact was or is to their life, using an 
11-point scale with a range from 0 (not at all disturbing) to 10 (very disturbing). Patient responses to discussion questions were captured by the moderator on a de-identified copy of the guide as well as on de-identified worksheets for reported symptoms and impacts. Interviews were audiorecorded with the participants' permission and interviewers took informal notes to keep track of the conversation topic at hand. Transcripts were not returned for comment or correction and no repeat interviews were conducted.

\subsection{Data Analysis}

Ahead of coding, a preliminary codebook was designed that captured all symptoms, signs, and impacts identified in the draft conceptual model. Two coders then grouped patient expressions from the de-identified audio-recorded interview transcripts by similar content for qualitative analysis using Atlas.ti ${ }^{\mathrm{TM}}$ software (version 8; ATLAS.ti Scientific Software Development GmbH, Berlin, Germany). Concepts were assigned a code that was disease related, treatment related, or both, depending on whether patients had attributed the concept to their CLL or its treatment, and whether they reported it occurring before, during, or after treatment. Instances where the patient was unsure of the symptom's cause were not coded. If a new concept was identified during coding, this triggered a project team meeting to assess if the evidence was sufficient to support its inclusion in the code book.

The two coders first coded the same transcripts independently for assessment of inter-coder agreement, and to discuss changes to the codebook and coding rules. Good intercoder agreement (predefined as Krippendorff's coefficient alpha binary $>0.7$ [25]) was reached after four transcripts, thus providing assurance of the consistency of coding and allowing division of the remaining transcripts among the coders. Periodic re-assessment of inter-coder agreement was interspersed throughout the coding process (spaced at predetermined regular intervals such that $25 \%$ of all transcripts were assessed by both coders), as were alignment meetings between coders to ensure the codebook remained up to date. Patient descriptions of concepts were reviewed for any distinct sub-components that should be highlighted.

Concept saturation was assessed separately for $1 \mathrm{~L}$ and $\mathrm{R} / \mathrm{R}$ by organizing transcripts chronologically into four groups of five transcripts each, and comparing concepts mentioned by patients in a group of interview transcripts with concepts mentioned in the previous group(s). If no new concepts appeared, saturation was achieved.

To identify the most salient concepts, the number of patients mentioning each concept and the concept's mean disturbance rating were mapped on a scatter plot separately for $1 \mathrm{~L}$ and R/R. A concept was deemed "salient" if at least seven patients mentioned the concept and it had a disturbance rating of 6 or higher, or if at least 13 patients mentioned the concept and it had a mean disturbance rating of 4 or higher.

\section{Results}

\subsection{Review of Literature, Patient Blogs, and Forums}

The literature searches yielded 854 publications; 17 of the publications were identified as highly relevant to the patient experience [26-42]. A review of these publications yielded 27 concepts considered to be related to CLL and/or its treatment.

Concepts considered related to the disease were fatigue/ tiredness, bruising, shortness of breath, swollen lymph nodes, night sweats, recurrent infections/illness, and weight loss. Fatigue/tiredness was the most prevalent concept. Concepts considered related to the treatment of CLL were cough, infusion reactions, tumor lysis syndrome, mouth irritation or soreness, blood in urine or stools, and weight gain. Constipation, diarrhea, nausea/vomiting, pain/discomfort, rash, and fever were considered to be related to both the disease and its treatment. Concepts identified as immediate impacts were appetite loss, decreased physical functioning, and insomnia/sleep disturbance, and general impacts were anxiety/worry, decreased ability to maintain social/familial role, decreased cognitive/emotional functioning, depression, and financial difficulties.

All of the concepts identified from the literature review, with the exception of constipation, were also identified from the patient blogs and forums. In addition, the patient blogs and forums revealed six further prevalent concepts: muscle/joint aches (considered to be disease related); headaches (treatment related); weakness (disease and treatment related); fear of death (immediate impact); stress; and uncertainty (both general impacts). No additional concepts were identified from the review of CLL treatment-related symptoms and adverse event details. A draft conceptual model was created from the review of the literature, patient blogs, and forums (Fig. 1).

\subsection{Interviews with Expert Clinicians}

The expert clinicians reported fatigue as the most prevalent concept among patients with symptomatic CLL, thereby confirming the findings from the literature review. It was noted by the clinicians that fatigue is multi-faceted, being related to both the feelings of tiredness, and the resulting 
impacts of needing to rest and limiting activities of daily living. Example clinician quotations were: "Patients will come in and say: 'I don't have enough energy", and "They tend to describe it in a functional way: 'I can't get up and make myself a cup of tea without feeling exhausted'". Recurrent infections and enlarged lymph nodes were reported by clinicians as key concepts in relation to CLL. Amendments to the draft conceptual model that were suggested by the clinicians are listed in the ESM 2.

\subsection{Qualitative Patient Interviews}

Qualitative interviews were conducted with 20 patients with $1 \mathrm{~L} \mathrm{CLL}$ and 20 patients with R/R CLL. The patients represented a wide range of demographic and clinical backgrounds (Table 1). Overall, patients with $1 \mathrm{~L}$ and R/R CLL were similar in terms of sex and age distributions. The time since diagnosis was longer in patients with $\mathrm{R} / \mathrm{R}$ than in those with $1 \mathrm{~L}$ disease, with $90 \%$ of patients with R/R CLL having been diagnosed at least 4 years previously, compared with $45 \%$ of those with $1 \mathrm{~L}$ disease. Clinician certification of diagnosis and disease staging was obtained from 14 patients (35\%); issues with clinician response and timing of office visits were cited as reasons for not being able to provide certification.

\subsubsection{Symptom Concepts}

The patient interviews revealed 35 symptom concepts, of which 28 were mentioned by patients with 1L CLL and 34 by patients with R/R CLL; 27 symptom concepts were mentioned by both patients with 1L CLL and those with R/R CLL. Symptom concept saturation grids for $1 \mathrm{~L}$ and R/R CLL are shown in the ESM 3 and 4, respectively. For 1L CLL, 11\% of the symptom concepts were mentioned in the last group of interview transcripts, and complete concept saturation was therefore not reached. However, no new symptoms were identified by the 20th interview. For R/R CLL, no new symptom concepts were mentioned in the last group of interview transcripts and concept saturation was therefore reached.

Figure 2 shows the number of patients mentioning symptoms spontaneously or when probed, together with the symptoms' mean disturbance ratings, for symptoms that were mentioned by at least four patients with $1 \mathrm{~L}$ or R/R CLL during the interviews. Fatigue was the most prominent and impactful symptom concept. It was mentioned by all patients with $1 \mathrm{~L}$ and R/R disease, and most (1L: 95\%; R/R: 85\%) mentioned it spontaneously. Although symptom patterns were generally similar between the $1 \mathrm{~L}$ and $\mathrm{R} / \mathrm{R}$ groups, a few symptoms were mentioned by considerably more patients with $R / R$ than with $1 \mathrm{~L}$ disease, including nausea/vomiting, fever, weight

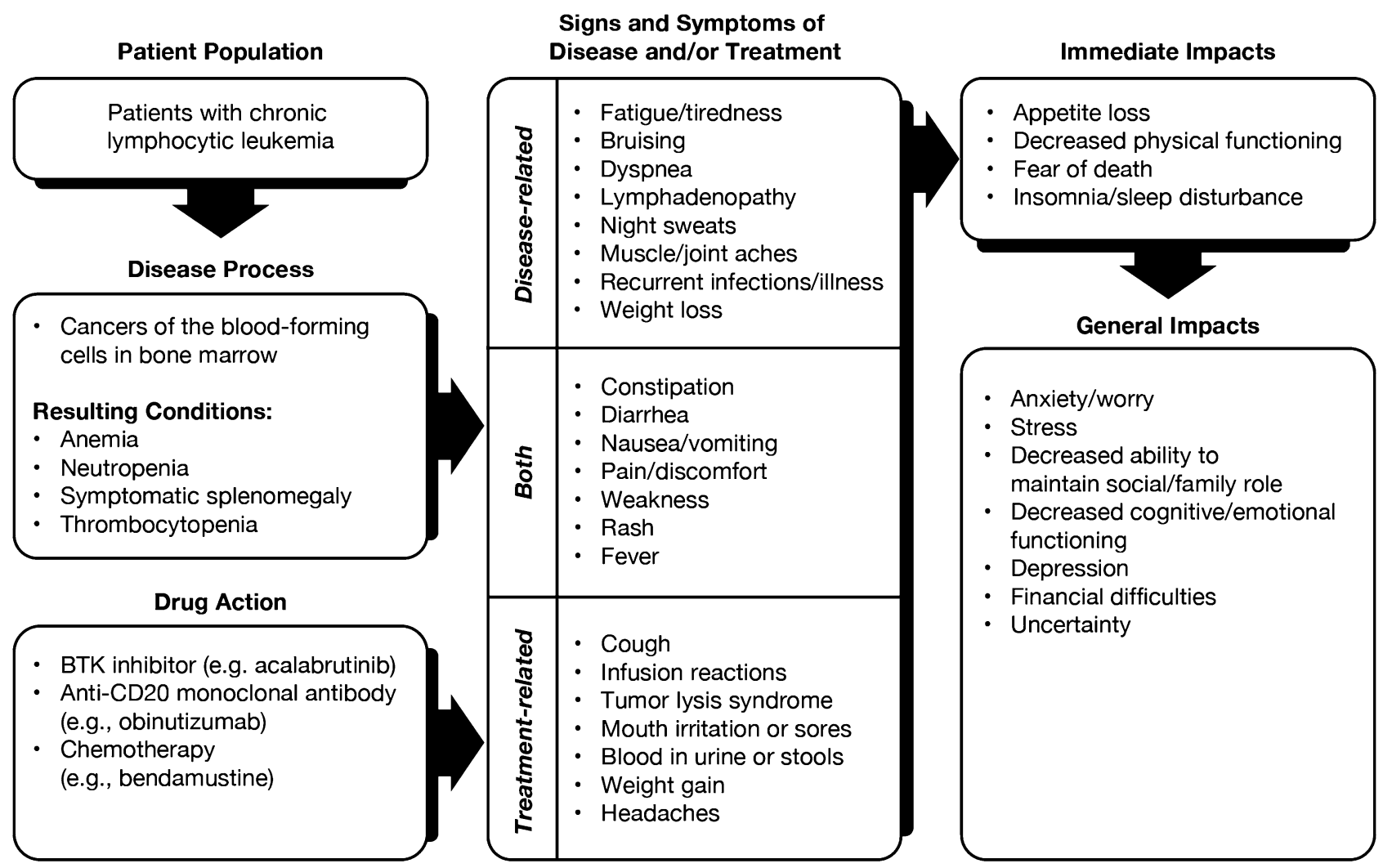

Fig. 1 Preliminary conceptual model, based on a review of the literature and patient blogs/forums. BTK Bruton tyrosine kinase 
Table 1 Summary demographics of patient interview respondents

\begin{tabular}{|c|c|c|}
\hline Demographic characteristic & $\begin{array}{l}\text { Patients with } \\
\text { 1L CLL } \\
(n=20)\end{array}$ & $\begin{array}{l}\text { Patients with } \\
\text { R/R CLL } \\
(n=20)\end{array}$ \\
\hline \multicolumn{3}{|l|}{ Age [years, $n(\%)$ ] } \\
\hline $18-35$ & $1(5)$ & $1(5)$ \\
\hline $36-50$ & $5(25)$ & $3(15)$ \\
\hline $51-65$ & $10(50)$ & $10(50)$ \\
\hline$\geq 66$ & $4(20)$ & $6(30)$ \\
\hline \multicolumn{3}{|l|}{$\operatorname{Sex}[n(\%)]$} \\
\hline Male & $10(50)$ & $11(55)$ \\
\hline Female & $10(50)$ & $9(45)$ \\
\hline \multicolumn{3}{|l|}{ US region $[n(\%)]$} \\
\hline Midwest & $4(20)$ & $4(20)$ \\
\hline Northeast & $6(30)$ & $3(15)$ \\
\hline Southeast & $8(40)$ & $5(25)$ \\
\hline Southwest & $2(10)$ & $3(15)$ \\
\hline West & $0(0)$ & $5(25)$ \\
\hline \multicolumn{3}{|l|}{ ECOG status $[n(\%)]$} \\
\hline 0 & $8(40)$ & $3(15)$ \\
\hline 1 & $6(30)$ & $10(50)$ \\
\hline 2 & $6(30)$ & $7(35)$ \\
\hline \multicolumn{3}{|c|}{ Time since diagnosis [years, $n(\%)$ ] } \\
\hline$<1$ & $2(10)$ & $0(0)$ \\
\hline $1-3$ & $9(45)$ & $2(10)$ \\
\hline $4-6$ & $5(25)$ & $6(30)$ \\
\hline$>6$ & $4(20)$ & $12(60)$ \\
\hline \multicolumn{3}{|c|}{ Time since most recent treatment [months, $n(\%)$ ] } \\
\hline NA (no treatment) & $13(65)$ & $0(0)$ \\
\hline$<6$ & $7(35)$ & $7(35)$ \\
\hline $6-12$ & $0(0)$ & $4(20)$ \\
\hline $1-3$ & $0(0)$ & $2(10)$ \\
\hline $4-6$ & $0(0)$ & $3(15)$ \\
\hline$>6$ & $0(0)$ & $4(20)$ \\
\hline
\end{tabular}

$1 L$ first-line, $C L L$ chronic lymphocytic leukemia, ECOG Eastern Cooperative Oncology Group, $N A$ not applicable, $R / R$ relapsed/refractory

loss, pain/discomfort, and infusion reactions. Symptoms with high mean disturbance ratings in both populations were fatigue and recurrent infections/illness. In addition, high disturbance ratings were obtained in patients with $1 \mathrm{~L}$ CLL for cough, weakness, fever, and weight gain, and in patients with $\mathrm{R} / \mathrm{R}$ CLL for sensitivity to bug bites and infusion reactions. Example quotations, from patients with R/R CLL were: "And I had been so sick with the flu, the $C$. diff[icile], it just went from one thing to the other. At the beginning of the year I think I had five emergency room visits or Urgent Care ... and then I could just not fight that flu, the $C$. diff, and I had a sinus infection" and "I've had fevers. Twice I was hospitalized with fever of unknown origin ... Because as the temperature was rising, my whole body seemed to be shutting down".
The symptom concepts mentioned by patients confirmed those identified from the literature and patient blog/forum reviews, and the clinician interviews. Three symptoms that were not part of the draft conceptual model were mentioned by at least four patients with $1 \mathrm{~L}$ or CLL disease: sensitivity to bug bites (1L: 6; R/R: 5); excessive bleeding (1L: 5; R/R: 6); and easily chipped nails (1L: 2; R/R: 4). These symptoms were added to the conceptual model. In addition, based on the patient interviews, changes were made to symptom attributions for bruising (revised from disease related to both disease and treatment related), and constipation, cough, headaches, and mouth irritation or soreness (revised from treatment related to both disease and treatment related).

\subsubsection{Impact Concepts}

The patient interviews revealed 16 impact concepts, of which 15 were mentioned by patients with $1 \mathrm{~L}$ CLL and all 16 were mentioned by patients with R/R CLL. Impact concept saturation grids for $1 \mathrm{~L}$ and $\mathrm{R} / \mathrm{R} \mathrm{CLL}$ are shown in ESM 5 and 6, respectively. For 1L CLL, 13 impacts were mentioned in the first group of interview transcripts, and no additional impacts were mentioned in the second and third groups of interview transcripts. However, one patient mentioned appetite gain in the last group of interviews. It was described by the patient as being caused by their depression and decreased physical functioning, and was thus not considered to be a new concept. Concept saturation was therefore considered to have been reached. For R/R CLL, all impact concepts were mentioned in the first group of interviews.

Figure 3 shows the number of patients mentioning impacts spontaneously or probed, together with the mean disturbance ratings, for impacts that were mentioned by at least four patients with $1 \mathrm{~L}$ or $\mathrm{R} / \mathrm{R}$ CLL during the interviews. Anxiety/worry was the most commonly reported impact in patients with 1L CLL. Decreased physical functioning was the most common impact in patients with R/R CLL, and was the most common spontaneously mentioned impact in both patient groups. Patterns were generally similar between patients with R/R and 1L CLL; however, considerably more patients with $\mathrm{R} / \mathrm{R}$ than $1 \mathrm{~L}$ CLL mentioned decreased cognitive/emotional functioning and fear of death. Impacts with high mean disturbance ratings in both $1 \mathrm{~L}$ and R/R CLL were: decreased physical functioning; decreased ability to maintain social/family role; and financial difficulties. In addition, high disturbance ratings were obtained in patients with 1L CLL for stress, insomnia, and decreased cognitive/ emotional functioning, and in patients with R/R CLL for depression. Example patient quotations were: "That is my worry as this does progress that I won't be able to enjoy their [children] lives as much as I would like to" (patient with 1L CLL) and "Not being able to walk a longer distance, walk upstairs, lift as much weight...picking up bags and moving 


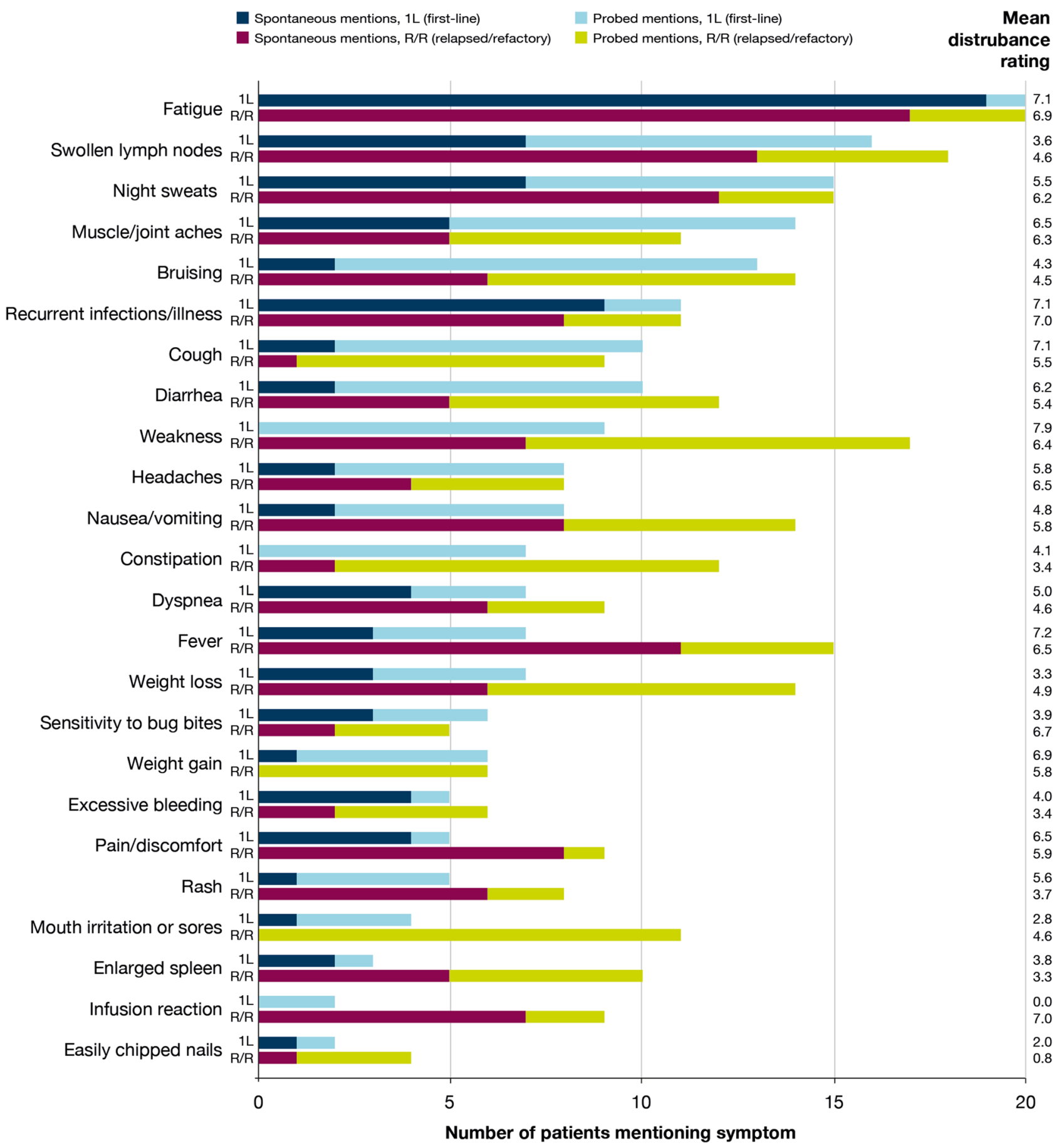

Fig. 2 Number of patients mentioning symptoms spontaneously or probed, and mean disturbance rating, for symptoms mentioned by four or more patients with first-line $(1 \mathrm{~L})$ or relapsed/refractory $(\mathrm{R} / \mathrm{R})$ chronic lymphocytic leukemia

tables or whatever. Just don't have the strength to do some of it" (patient with R/R CLL).

The impact concepts mentioned by patients confirmed those identified from the literature and patient blog/forum reviews, and the clinician interviews. Two impacts that were not part of the draft conceptual model were mentioned by at least four patients with 1L or CLL disease: decreased ability to work (1L: $11 ; \mathrm{R} / \mathrm{R}: 15)$ and more cautious behavior (1L: 7; R/R: 6). These impacts were added to the conceptual model. "Appetite loss", which clinicians had suggested rewording as "early satiety" (ESM 2), was revised back to "appetite loss" based on the patients' descriptions. 


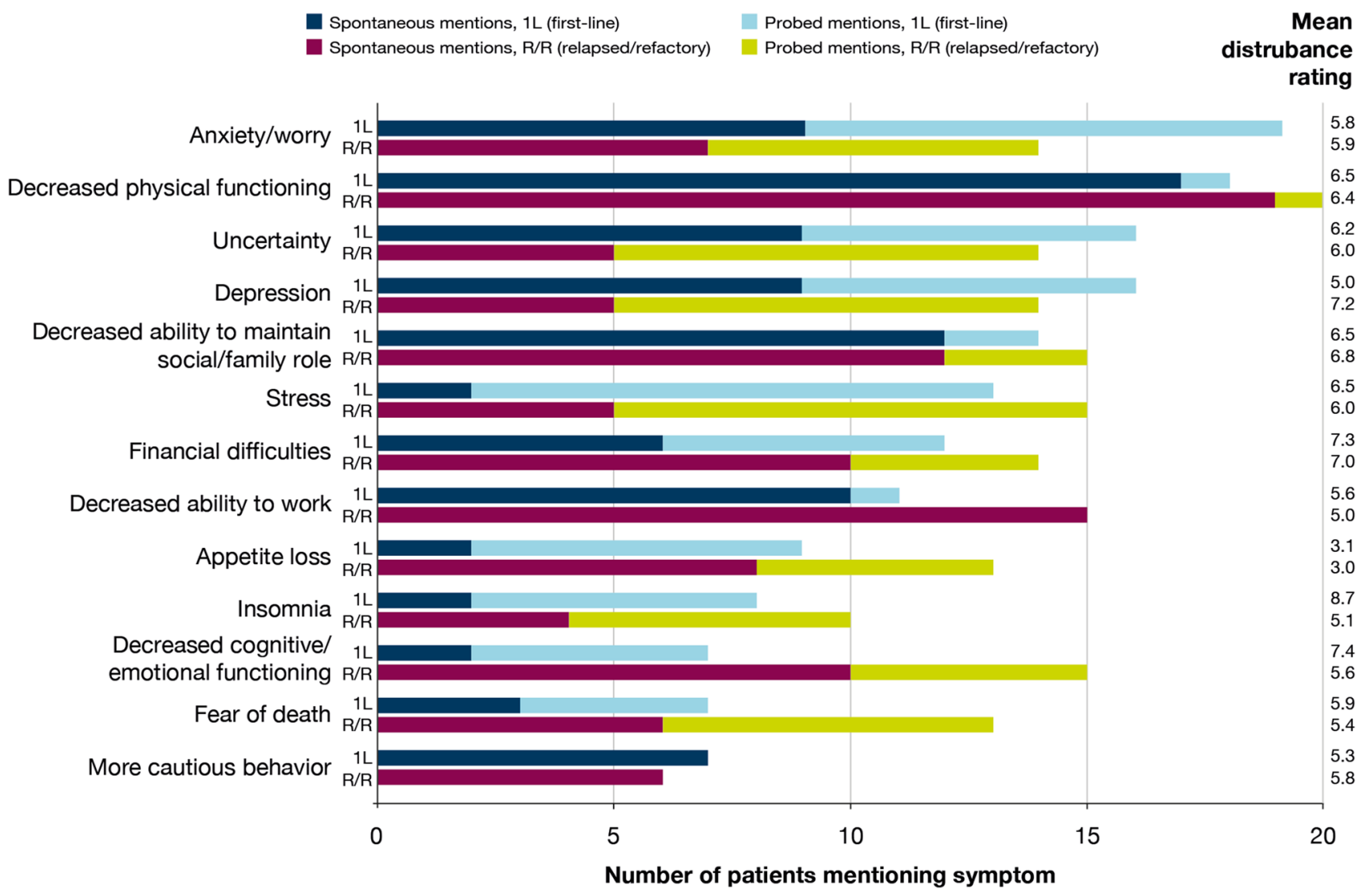

Fig. 3 Number of patients mentioning impacts spontaneously or probed, and mean disturbance rating, for impacts mentioned by four or more patients with first-line (1L) or relapsed/refractory $(\mathrm{R} / \mathrm{R})$ chronic lymphocytic leukemia

\subsection{Fatigue-Related Sub-Components}

From how patients described their fatigue, it was apparent that it manifests as a variety of symptoms and impacts. Fatigue-related symptoms were: tiredness/need for sleep; lack of energy; weakness; and cognitive fatigue. In addition, three of the impact concepts were identified as fatiguerelated sub-components based on how patients described fatigue; these were: decreased ability to maintain social, familial or professional role; decreased physical functioning; and frustration.

The number of patients mentioning each fatigue-related sub-component, and examples of patient quotations are listed in Table 2. Patients described their fatigue-related symptoms as: being worn out more quickly by normal activities; having a strong need to nap during the day even if not exerting oneself; lacking motivation to move or be active; being unable to push oneself to do desired activities; difficulty carrying out normal activities; inability to exercise normally; difficulty moving around or doing chores; inability to focus; and difficulty concentrating for long periods. Patients described their fatigue-related impacts as: a reduced ability to socialize with friends; partners needing to take on more familial responsibilities; ability to work suffering or being lost entirely; normal activities becoming more difficult; certain tasks no longer being feasible; and being frustrated at the lack of energy or ability to do what they used to be able to do. The conceptual model was updated to include the fatigue-related symptom and impact sub-components.

\subsection{Conceptual Model}

Three versions of the conceptual model were created: an overall model with all identified concepts, and two models highlighting the most salient concepts in $1 \mathrm{~L}$ and R/R (Fig. 4). Muscle/joint aches, night sweats, bruising, fatigue-related symptoms, fever, and recurrent infections/illness were salient symptoms in both $1 \mathrm{~L}$ and R/R. Salient impacts in both $1 \mathrm{~L}$ and $R / R$ were fatigue-related impacts, insomnia, uncertainty, decreased cognitive/emotional functioning, anxiety/worry, stress, depression, financial difficulty, and fear of death. Dyspnea and cough were salient in $1 \mathrm{~L}$ only, and enlarged lymph nodes, headaches, pain/discomfort, weight loss, nausea/vomiting, and infusion reactions were salient in R/R only. 
Table 2 Fatigue-related symptom and impact sub-components, and example patient quotations

\begin{tabular}{|c|c|c|c|}
\hline \multirow[t]{2}{*}{ Fatigue-related sub-component } & \multicolumn{2}{|c|}{$\begin{array}{l}\text { Patients } \\
(n) \text { men- } \\
\text { tioning }\end{array}$} & \multirow[t]{2}{*}{ Example quotation } \\
\hline & $1 \mathrm{~L}$ & $\mathrm{R} / \mathrm{R}$ & \\
\hline \multicolumn{4}{|l|}{ Fatigue-related symptom } \\
\hline Tiredness/need to sleep & 15 & 17 & $\begin{array}{l}\text { "I got a lot tired, a lot more tired. I just, I didn't do much during the day. Almost all I could do } \\
\text { would be to wake up, eat breakfast, go back to bed. Wake up, eat dinner, go back to bed" }\end{array}$ \\
\hline Lack of energy & 13 & 15 & $\begin{array}{l}\text { "I had a hard time accomplishing a day's long series of tasks that I had been used to doing without } \\
\text { just absolutely collapsing in a chair, and a feeling of very washed out, just not having the energy } \\
\text { to do things" }\end{array}$ \\
\hline Weakness $^{\mathrm{a}}$ & 3 & 1 & $\begin{array}{l}\text { "I remember [I used] to carry a case of water in the house. That's something that should be easy } \\
\text { for me. But I would rather have my daughter come over and carry it in because I just sometimes } \\
\text { feel weak" }\end{array}$ \\
\hline Cognitive fatigue $^{a}$ & 2 & 7 & $\begin{array}{l}\text { "It's kind of like a foggy feeling. I can't really think straight, and I'm just really super tired, almost } \\
\text { like [after] a big Thanksgiving meal" }\end{array}$ \\
\hline \multicolumn{4}{|l|}{ Fatigue-related impact } \\
\hline $\begin{array}{l}\text { Decreased ability to maintain } \\
\text { social/familial/professional } \\
\text { role }^{\mathrm{a}}\end{array}$ & 11 & 12 & $\begin{array}{l}\text { "I don't see my friends anymore because if I make plans they end up getting cancelled because I'm } \\
\text { too tired or I don't feel good. I have the best intentions to make it, but I usually can't, and that } \\
\text { happens with all my family members" }\end{array}$ \\
\hline Decreased physical functioning ${ }^{a}$ & 17 & 17 & $\begin{array}{l}\text { "I realized I couldn't do what I used to do ... any type of real exertion, I couldn't do ... [I used to] } \\
\text { dance. I used to play volleyball with my grandkids and [I] can't do any of those things anymore } \\
\ldots \text { today, I feel like today I'm afraid to even go out and try to walk because I never know when } \\
\text { that real tired feeling is going to hit me" }\end{array}$ \\
\hline Frustration & 8 & 13 & "I'm tired. I feel so tired. I sleep all the time. I sleep horrible. It's real irritating" \\
\hline
\end{tabular}

${ }^{a}$ Total number of mentions relates to when a patient, unprompted, used the sub-component to describe their fatigue

\section{Discussion}

In this qualitative study, a conceptual model of CLL was created that captures disease- and treatment-related symptoms, signs, and impacts in patients with $1 \mathrm{~L}$ or R/R CLL. Data from interviews with clinical experts and patients with CLL provided support of the validity of concepts identified from the review of the literature and patient blogs/forums, and identified additional concepts that were not captured in the review. Although the $1 \mathrm{~L}$ and $\mathrm{R} / \mathrm{R}$ groups mentioned mostly the same symptom, sign, and impact concepts, for several concepts, there were differences between the $1 \mathrm{~L}$ and $\mathrm{R} / \mathrm{R}$ groups in the number of patients mentioning the concept, as well as in its mean disturbance rating. Thus, in addition to an overall conceptual model with all identified concepts, two additional models were developed that separately highlight the most salient concepts in $1 \mathrm{~L}$ and $\mathrm{R} / \mathrm{R}$ settings.

Qualitative work supports the development of new PRO instruments and is also necessary to assess the validity and comprehensiveness of existing instruments. In recent years, the shift towards continuous targeted therapies for CLL has provided the potential for long-term disease control. It is important to understand how patients experience and are affected by CLL and its treatment, particularly in the context of the chronic nature of both the disease and targeted therapies. The results of the current study provide an understanding of what might be important to measure in patients with $1 \mathrm{~L}$ or $\mathrm{R} / \mathrm{R} \mathrm{CLL}$. This knowledge enables researchers to determine whether a PRO instrument is the optimal tool for a concept to be measured, and which PRO instrument provides the most comprehensive capture of the patient experience, by mapping the instrument to the conceptual model. In addition, the different versions of the conceptual model developed in the current study can help to identify concepts from available PRO instruments that are unlikely to be relevant to patients with $1 \mathrm{~L}$ or R/R CLL.

The thresholds used to define concepts as salient in the current study (i.e., mentioned by $\geq 65 \%$ of patients and mean disturbance rating $\geq 4$; or mentioned by $\geq 35 \%$ of patients and mean disturbance rating $\geq 6$ ) allowed for "common and somewhat disturbing" as well as "less common but highly disturbing" concepts to be recognized in the conceptual model. There were notable differences between patients with $1 \mathrm{~L}$ and $\mathrm{R} / \mathrm{R}$ disease regarding the symptoms, signs, and impacts that were most salient. Infusion reactions and nausea/vomiting were treatment-related symptoms that were salient only in patients with R/R CLL. Their lower relevance in patients with $1 \mathrm{~L} \mathrm{CLL}$ can be explained by two-thirds $(13 / 20)$ of the patients with $1 \mathrm{~L}$ disease having been treatment naïve at the time of the qualitative interviews. Patients with early-stage CLL are commonly monitored without therapy ("watch and wait"), particularly if the symptom burden 


\begin{tabular}{|c|c|c|c|}
\hline Patient Population & \multicolumn{2}{|r|}{$\begin{array}{l}\text { Signs and Symptoms of } \\
\text { Disease and/or Treatment }\end{array}$} & Immediate Impacts \\
\hline $\begin{array}{l}\text { Patients with chronic } \\
\text { lymphocytic leukemia } \\
\text { Disease Process }\end{array}$ & 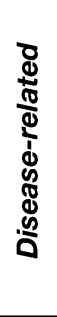 & $\begin{array}{l}\text { - Short of breath/out of } \\
\text { breath (1L) } \\
\text { - Swollen lymph nodes } \\
\text { (R/R) } \\
\text { - Night sweats (1L; R/R) } \\
\text { - Muscle/joint aches } \\
\text { (1L; R/R) } \\
\text { - Sensitivity to bug bites }\end{array}$ & $\begin{array}{l}\text { - Fatigue-related immediate } \\
\text { impacts (1L; R/R) } \\
\text { - Decreased physical } \\
\text { functioning } \\
\text { - Insomnia/sleep disturbance } \\
\text { (1L; R/R) } \\
\text { - Uncertainty (1L; R/R) } \\
\text { - Limitations in activities of } \\
\text { daily living: need for rest }\end{array}$ \\
\hline $\begin{array}{l}\text { Resulting Conditions: } \\
\text { - Anemia } \\
\text { - Neutropenia } \\
\text { - Enlarged spleen } \\
\text { - Thrombocytopenia } \\
\text { - Tumor lysis sundrome }\end{array}$ & ई & $\begin{array}{l}\text { - Pain/discomfort (R/R) } \\
\text { - Rash } \\
\text { - Fever (1L; R/R) } \\
\text { - Fatigue symptoms } \\
\text { (1L; R/R) } \\
\text { - Tiredness/need sleep } \\
\text { - Weakness } \\
\text { - Lack of energy } \\
\text { - Cognitive fatigue } \\
\text { - Recurrent infections/ } \\
\text { illness (1L; R/R) } \\
\text { - Weight loss (R/R) } \\
\text { - Bruising (1L; R/R) } \\
\text { - Constipation } \\
\text { - Cough (1L) } \\
\text { - Headaches (R/R) } \\
\text { - Mouth irritation or sores } \\
\text { - Excessive bleeding }\end{array}$ & $\begin{array}{l}\text { General Impacts } \\
\text { - Fatigue-related general } \\
\text { impacts (1L; R/R) } \\
\text { - Decreased ability to } \\
\text { maintain social/familial/ } \\
\text { professional role } \\
\text { - Frustration } \\
\text { - Decreased cognitive/ } \\
\text { emotional functioning (1L; R/R) } \\
\text { - Anxiety/worry (1L; R/R) } \\
\text { - Stress (1L; R/R) } \\
\text { - Depression (1L; R/R) } \\
\text { - Financial difficulties (1L; R/R) } \\
\text { - Fear of death (1L; R/R) } \\
\text { - Appetite loss }\end{array}$ \\
\hline $\begin{array}{l}\text { BTK inhibitor (e.g. acalabrutinib) } \\
\text { - Anti-CD20 monoclonal antibody } \\
\text { (e.g., obinutizumab) } \\
\text { - Chemotherapy } \\
\text { (e.g., bendamustine) }\end{array}$ & $\frac{\substack{0 \\
0 \\
\frac{\pi}{0}}}{\frac{1}{0}}$ & $\begin{array}{l}\text { - Blood in urine or stools } \\
\text { - Weight gain } \\
\text { - Infusion reactions (R/R) } \\
\text { - Diarrhea } \\
\text { - Nausea/vomiting (R/R) } \\
\text { - No appetite/feeling full } \\
\text { - Easily chipped nails }\end{array}$ & - More cautious behavior \\
\hline
\end{tabular}

Fig. 4 Chronic lymphocytic leukemia conceptual model. Bold text indicates the most salient concepts in first-line (1L) and/or relapsed/refractory $(\mathrm{R} / \mathrm{R})$ disease. BTK Bruton tyrosine kinase

is low and there is no evidence of disease progression [2]. Other concepts that were salient only in patients with $R / R$ disease were swollen lymph nodes, pain/discomfort, weight loss, and headache. Unintentional weight loss, pain/discomfort, and headache were attributed to both the disease and its treatment. Large swollen lymph nodes can be a sign of progressive disease [2].

Fatigue was a key issue mentioned by all patients. This finding should be contextualized by the study eligibility criteria, which required patients to have experienced at least one of four constitutive symptoms of CLL (fatigue, weight loss, fever, or night sweats) in the week before study entry (all patients indicated they had fatigue). However, fatigue/ tiredness was also the key symptom reported in another study, which assessed the feasibility of using online surveys to elicit concepts relevant to CLL [34]. Although being symptomatic was not part of that study's eligibility criteria, $78 \%$ of patients reported experiencing at least one symptom, and $54 \%$ of patients mentioned experiencing fatigue/tiredness. In follow-up telephone interviews, patients expressed additional symptom and impact concepts (e.g., cognitive difficulties and difficulty staying asleep) that the same patients had not reported in the online survey, highlighting the superiority of traditional qualitative interview approaches for comprehensiveness when eliciting patients' experiences.

From how patients in the current study described fatiguerelated symptoms and impacts, it was apparent that the concept was multi-faceted and comprised a variety of subcomponents. Fatigue manifested in a range of symptoms (tiredness/need for sleep, lack of energy, weakness, cognitive fatigue) and resultant impacts (decreased ability to maintain social/familial/professional role, decreased physical 
functioning, frustration). The description of fatigue in the conceptual model was thus expanded to include the fatiguerelated sub-components. All fatigue-related symptom and impact sub-components were found to be salient both for patients with $1 \mathrm{~L}$ and those with R/R CLL. In the current study, there were also other highly disturbing symptoms or impacts beyond fatigue that were commonly mentioned by both patients with $1 \mathrm{~L}$ and those with $\mathrm{R} / \mathrm{R}$ disease, including night sweats, muscle/joint aches, fever, recurrent infections/ illness, insomnia/sleep disturbance, uncertainty, and other, more general impacts (anxiety/worry, stress, depression, financial difficulties, fear of death).

The current work contributes valuable evidence of early patient input and is the foundation for valid comprehensive PRO instruments being used in future clinical treatment trials in patients with CLL. It provides an understanding of how patients with CLL are affected by their disease and, through this knowledge of what is important to patients, can guide the development of PRO endpoints and reinforce the importance of their inclusion in clinical trials. Collecting evidence on the patient experience will help to articulate clearly to patients what to expect from treatment, in a manner relevant to their experience. If patients know what to expect from treatment, from the point of view of other patients who have already received it, they can make informed treatment decisions. This information could also aid effective dialogue between patients and clinicians.

This work had several important strengths. It was based on rigorous qualitative methodologies that identified concepts considered to be related to CLL and/or its treatment based on searches of the literature and patient blogs/forums, and qualitative interviews with expert clinicians and patients with CLL. Separate sub-analyses were conducted for $1 \mathrm{~L}$ and $\mathrm{R} / \mathrm{R}$ CLL. Limitations include that clinician certification of diagnosis and disease staging was obtained from 35\% of patients only, and that full concept saturation was not reached for 1L CLL. The study included only patients who had at least one of the constitutional symptoms of CLL in the week before study entry; this approach ensured that all included patients could contribute to the understanding of the signs, symptoms, and impacts of CLL, although it did exclude the experiences of patients with CLL who were non-symptomatic.

\section{Conclusions}

Three versions of a conceptual model of CLL were developed to capture salient symptoms in patients with $1 \mathrm{~L}$ or $\mathrm{R} / \mathrm{R}$ disease. Fatigue was identified as the key issue affecting patients with CLL. The comprehensive conceptual model of the patient experience with CLL was developed from direct inputs from patients rather than from existing PRO instruments used in clinical studies, which are limited to capturing what is assumed to be appropriate to capture. The conceptual model is a resource to aid appropriate selection and content validation of PRO instruments for future clinical trials and may also help to identify concepts that are unlikely to be relevant. The conceptual model can help inform patient-centered endpoints to evaluate new treatments for $1 \mathrm{~L}$ and/or R/R CLL.

Acknowledgement The authors thank O. Meyers and S. Lee, of IQVIA, New York, NY, USA, for their participation in conducting the interviews.

Author Contributions DE, HC, and TAE: conceptualized and designed the study; MB, CK, and TAE: collected the data. MB and CK: analyzed the data; DE, MB, CK, HC, and TAE: interpreted the results and substantively revised the manuscript. All authors approved the final version and are accountable for all aspects of the work.

\section{Declarations}

Funding This qualitative study was funded by AstraZeneca. Medical writing support was provided by Anja Becher, $\mathrm{PhD}$, of Oxford PharmaGenesis, Oxford, UK, and was funded by AstraZeneca.

Conflict of Interest Daniel Eek and Helena Chung are employees of AstraZeneca and hold shares in AstraZeneca. Matthew Blowfield and Calvin Krogh are employees of IQVIA, which received funds from AstraZeneca to conduct the study. Toby A. Eyre has received honoraria from Roche, Gilead, Janssen, AbbVie, and AstraZeneca, research support from Gilead, and travel support to scientific conferences from Gilead and AbbVie.

Ethics Approval The patient interviews were approved by the New England Institutional Review Board.

Consent to Participate All interviewed patients completed an informed consent form

Consent for Publication Not applicable.

Availability of Data and Material Acerta Pharma, a member of the AstraZeneca Group, is committed to data transparency and will consider data sharing requests on a case-by-case basis. Any requests for de-identified patient data can be submitted to Acerta Pharma 3 months post-publication and ending 5 years following article publication with the intent-to-achieve aims of the original proposal. In addition, Acerta Pharma will provide the study protocol, statistical analysis plan, and informed consent form, as well as post results on clinicaltrials.gov, as required.

Code Availability Not applicable.

Open Access This article is licensed under a Creative Commons Attribution-NonCommercial 4.0 International License, which permits any non-commercial use, sharing, adaptation, distribution and reproduction in any medium or format, as long as you give appropriate credit to the original author(s) and the source, provide a link to the Creative Commons licence, and indicate if changes were made. The images or other third party material in this article are included in the article's Creative Commons licence, unless indicated otherwise in a credit line to the material. If material is not included in the article's Creative Commons 
licence and your intended use is not permitted by statutory regulation or exceeds the permitted use, you will need to obtain permission directly from the copyright holder. To view a copy of this licence, visit http://creativecommons.org/licenses/by-nc/4.0/.

\section{References}

1. Hallek M. Chronic lymphocytic leukemia: 2017 update on diagnosis, risk stratification, and treatment. Am J Hematol. 2017;92(9):946-65.

2. Hallek M, Cheson BD, Catovsky D, Caligaris-Cappio F, Dighiero $\mathrm{G}$, Dohner H, et al. iwCLL guidelines for diagnosis, indications for treatment, response assessment, and supportive management of CLL. Blood. 2018;131(25):2745-60.

3. von Tresckow J, Eichhorst B, Bahlo J, Hallek M. The treatment of chronic lymphatic leukemia. Dtsch Arztebl Int. 2019;116(4):41-6.

4. Byrd JC, Brown JR, O'Brien S, Barrientos JC, Kay NE, Reddy $\mathrm{NM}$, et al. Ibrutinib versus ofatumumab in previously treated chronic lymphoid leukemia. N Engl J Med. 2014;371(3):213-23.

5. Burger JA, Tedeschi A, Barr PM, Robak T, Owen C, Ghia P, et al. Ibrutinib as initial therapy for patients with chronic lymphocytic leukemia. N Engl J Med. 2015;373(25):2425-37.

6. Jones JA, Robak T, Brown JR, Awan FT, Badoux X, Coutre S, et al. Efficacy and safety of idelalisib in combination with of ofatumumab for previously treated chronic lymphocytic leukaemia: an open-label, randomised phase 3 trial. Lancet Haematol. 2017;4(3):e114-26.

7. Furman RR, Sharman JP, Coutre SE, Cheson BD, Pagel JM, Hillmen $P$, et al. Idelalisib and rituximab in relapsed chronic lymphocytic leukemia. N Engl J Med. 2014;370(11):997-1007.

8. Fischer K, Al-Sawaf O, Bahlo J, Fink AM, Tandon M, Dixon M, et al. Venetoclax and obinutuzumab in patients with CLL and coexisting conditions. N Engl J Med. 2019;380(23):2225-36.

9. Thanarajasingam G, Minasian LM, Baron F, Cavalli F, De Claro RA, Dueck AC, et al. Beyond maximum grade: modernising the assessment and reporting of adverse events in haematological malignancies. Lancet Haematol. 2018;5(11):e563-98.

10. Basch E, Deal AM, Kris MG, Scher HI, Hudis CA, Sabbatini P, et al. Symptom monitoring with patient-reported outcomes during routine cancer treatment: a randomized controlled trial. J Clin Oncol. 2016;34(6):557-65.

11. EMA. Reflection paper on the regulatory guidance for the use of health-related quality of life (HRQL) measures in the evaluation of medicinal products. 2004. https://www.ema.europa.eu/ en/documents/scientific-guideline/reflection-paper-regulatory -guidance-use-healthrelated-quality-life-hrql-measures-evalu ation_en.pdf. Accessed 12 Nov 2019.

12. EMA. Reflection paper on the use of patient reported outcome (PRO) measures in oncology studies. 2014. https://www.ema. europa.eu/en/documents/presentation/presentation-reflection -paper-use-patient-reported-outcome-pro-measures-oncologystudies-daniel_en.pdf. Accessed 12 Nov 2019.

13. US FDA. Patient-reported outcome measures: use in medical product development to support labeling claims. 2009. https:// www.fda.gov/regulatory-information/search-fda-guidance-docum ents/patient-reported-outcome-measures-use-medical-productdevelopment-support-labeling-claims. Accessed 12 Nov 2019.

14. Efficace F, Fayers P, Pusic A, Cemal Y, Yanagawa J, Jacobs M, et al. Quality of patient-reported outcome reporting across cancer randomized controlled trials according to the CONSORT patientreported outcome extension: a pooled analysis of 557 trials. Cancer. 2015;121(18):3335-42.

15. Kyte D, Retzer A, Ahmed K, Keeley T, Armes J, Brown JM, et al. Systematic evaluation of patient-reported outcome protocol content and reporting in cancer trials. J Natl Cancer Inst. 2019;111(11):1170-8.

16. Retzer A, Keeley T, Ahmed K, Armes J, Brown JM, Calman L, et al. Evaluation of patient-reported outcome protocol content and reporting in UK cancer clinical trials: the EPiC study qualitative protocol. BMJ Open. 2018;8(2):e017282.

17. Basch E. Toward patient-centered drug development in oncology. N Engl J Med. 2013;369(5):397-400.

18. van de Poll-Franse L, Oerlemans S, Bredart A, Kyriakou C, Sztankay M, Pallua S, et al. International development of four EORTC disease-specific quality of life questionnaires for patients with Hodgkin lymphoma, high- and low-grade non-Hodgkin lymphoma and chronic lymphocytic leukaemia. Qual Life Res. 2018;27(2):333-45.

19. Robak T, Warzocha K, Babu KG, Kulyaba Y, Kuliczkowski K, Abdulkadyrov K, et al. Health-related quality of life and patientreported outcomes of ofatumumab plus fludarabine and cyclophosphamide versus fludarabine and cyclophosphamide in the COMPLEMENT 2 trial of patients with relapsed CLL. Leuk Lymphoma. 2017;58(7):1598-606.

20. Cramer P, Fraser G, Santucci-Silva R, Grosicki S, Dilhuydy MS, Janssens A, et al. Improvement of fatigue, physical functioning, and well-being among patients with severe impairment at baseline receiving ibrutinib in combination with bendamustine and rituximab for relapsed chronic lymphocytic leukemia/small lymphocytic lymphoma in the HELIOS study. Leuk Lymphoma. 2018;59(9):2075-84.

21. Hillmen P, Janssens A, Babu KG, Kloczko J, Grosicki S, Manson $\mathrm{S}$, et al. Health-related quality of life and patient-reported outcomes of ofatumumab plus chlorambucil versus chlorambucil monotherapy in the COMPLEMENT 1 trial of patients with previously untreated CLL. Acta Oncol. 2016;55(9-10):1115-20.

22. Barrientos JC, O’Brien S, Brown JR, Kay NE, Reddy NM, Coutre $\mathrm{S}$, et al. Improvement in parameters of hematologic and immunologic function and patient well-being in the phase III RESONATE study of ibrutinib versus ofatumumab in patients with previously treated chronic lymphocytic leukemia/small lymphocytic lymphoma. Clin Lymphoma Myeloma Leuk. 2018;18(12):803-13.

23. Liberati A, Altman DG, Tetzlaff J, Mulrow C, Gotzsche PC, Ioannidis JP, et al. The PRISMA statement for reporting systematic reviews and meta-analyses of studies that evaluate healthcare interventions: explanation and elaboration. BMJ. 2009;339:b2700.

24. Patrick DL, Burke LB, Gwaltney CJ, Leidy NK, Martin ML, Molsen E, et al. Content validity: establishing and reporting the evidence in newly developed patient-reported outcomes (PRO) instruments for medical product evaluation: ISPOR PRO Good Research Practices Task Force report: part 2: assessing respondent understanding. Value Health. 2011;14(8):978-88.

25. Krippendorff K. Content analysis: an introduction to its methodology. Beverly Hills: Sage Publications; 1980.

26. Eichhorst B, Goede V, Hallek M. Treatment of elderly patients with chronic lymphocytic leukemia. Leuk Lymphoma. 2009;50(2):171-8.

27. Else M, Smith AG, Cocks K, Richards SM, Crofts S, Wade R, et al. Patients' experience of chronic lymphocytic leukaemia: baseline health-related quality of life results from the LRF CLL4 trial. Br J Haematol. 2008;143(5):690-7.

28. Evans J, Ziebland S, Pettitt AR. Incurable, invisible and inconclusive: watchful waiting for chronic lymphocytic leukaemia and implications for doctor-patient communication. Eur J Cancer Care (Engl). 2012;21(1):67-77.

29. Frey S, Blankart CR, Stargardt T. Economic burden and qualityof-life effects of chronic lymphocytic leukemia: a systematic review of the literature. Pharmacoeconomics. 2016;34(5):479-98. 
30. Hus I, Rolinski J. Current concepts in diagnosis and treatment of chronic lymphocytic leukemia. Contemp Oncol (Pozn). 2015;19(5):361-7.

31. Jain P, Keating M, Renner S, Cleeland C, Xuelin H, Gonzalez GN, et al. Ruxolitinib for symptom control in patients with chronic lymphocytic leukaemia: a single-group, phase 2 trial. Lancet Haematol. 2017;4(2):e67-74.

32. Kutsch N, Busch R, Bahlo J, Mayer J, Hensel M, Hopfinger $\mathrm{G}$, et al. FCR front-line therapy and quality of life in patients with chronic lymphocytic leukemia. Leuk Lymphoma. 2017;58(2):399-407.

33. Levin TT, Li Y, Riskind J, Rai K. Depression, anxiety and quality of life in a chronic lymphocytic leukemia cohort. Gen Hosp Psychiatry. 2007;29(3):251-6.

34. McCarrier KP, Bull S, Fleming S, Simacek K, Wicks P, Cella $\mathrm{D}$, et al. Concept elicitation within patient-powered research networks: a feasibility study in chronic lymphocytic leukemia. Value Health. 2016;19(1):42-52.

35. Molica S. Quality of life in chronic lymphocytic leukemia: a neglected issue. Leuk Lymphoma. 2005;46(12):1709-14.

36. Olsson C, Sandin-Bojo AK, Bjuresater K, Larsson M. Changes in sexuality, body image and health related quality of life in patients treated for hematologic malignancies: a longitudinal study. Sex Disabil. 2016;34(4):367-88.

37. Pashos CL, Flowers CR, Kay NE, Weiss M, Lamanna N, Farber $\mathrm{C}$, et al. Association of health-related quality of life with gender in patients with B-cell chronic lymphocytic leukemia. Support Care Cancer. 2013;21(10):2853-60.

38. Pinilla-Ibarz J, Emole J. Chronic lymphocytic leukemia in the elderly, which investigations are necessary: a map for the practicing oncologist. Cancer Control. 2015;22(4 Suppl.):7-16.

39. Shanafelt TD, Bowen D, Venkat C, Slager SL, Zent CS, Kay NE, et al. Quality of life in chronic lymphocytic leukemia: an international survey of 1482 patients. Br J Haematol. 2007;139(2):255-64.

40. Shanafelt TD, Bowen DA, Venkat C, Slager SL, Zent CS, Kay $\mathrm{NE}$, et al. The physician-patient relationship and quality of life: lessons from chronic lymphocytic leukemia. Leuk Res. 2009;33(2):263-70.

41. Westbrook TD, Maddocks K, Andersen BL. The relation of illness perceptions to stress, depression, and fatigue in patients with chronic lymphocytic leukaemia. Psychol Health. 2016;31(7):891-902.

42. Morrison EJP. Psychological distress and symptom burden: vulnerabilities in chronic lymphocytic leukemia patients. Dissertation presented in partial fulfillment of the requirements for the degree of doctor of philosophy in the Graduate School of the Ohio State University. 2013. https://etd.ohiolink.edu/!etd.send_file?acces sion $=$ osu $1366305005 \&$ disposition $=$ inline. Accessed 12 Nov 2019. 